\title{
An Image Edge Detection Algorithm Based on Improved Wavelet Transform
}

\author{
Xumin Liu ${ }^{1}$, Zilong Duan ${ }^{1}$, Xiaojun Wang ${ }^{1}$ and Weixiang $\mathrm{Xu}^{2}$ \\ ${ }^{1}$ College of Information Engineering \\ Capital Normal University, Beijing 100048, P. R. China \\ ${ }^{2}$ School of Traffic and Transportation \\ Beijing Jiaotong University, Beijing 100044, P.R. China \\ liuxumin@126.com
}

\begin{abstract}
In this paper, for wavelet modulus maxima edge detection affected by noise largely, we propose an improved image fusion algorithm. Firstly, wavelet denoising and smoothing filter are conducted on the original image. Secondly, making edge detection with wavelet transform modulus maxima edge detection and improved canny edge detection get each of edge detection images. Thirdly, let the wavelet fusion follow some certain fusion rules. Finally, making the inverse wavelet transform reconstruct the fused image. The experimental results show that the fused image combines the advantages of edge detection methods, which is an effective image edge detection method.
\end{abstract}

Keywords: Wavelet modulus maxima edge detection; Wavelet fusion; Traditional Canny operator; Iterative algorithm; Mathematical Morphology

\section{Introduction}

For decades, although plenty of experts and scholars are striving for the great achievement of image edge detection technology [1] in algorithm and application areas, there also exist some corresponding problems, such as, image under actual processing is affected by noise, and the distinction between adjacent pixel gray values becomes smaller during the development of high resolution imaging techniques[2-3]. Those changes raise higher requirements on image detection, and the traditional algorithms are hard to satisfy these high requirements, thus promoting the research on image edge detection algorithm to deeper and wider direction. Besides, the research contents are enriched through the rapid development of science, and new theories and knowledge, providing the algorithm studies with a solid foundation to develop[4].

Compared with traditional Canny operator [7], wavelet modulus maximum edge detection algorithm [5-6] does well in edge detection, but is easily affected by noise to some extent. This paper provides an improved algorithm based on wavelet modulus maximum edge detection algorithm, which combines wavelet modulus maximum edge detection algorithm with improved Canny algorithm by wavelet fusion technology. The final edge detection image is gained by refactoring fusion image with wavelet inverse translation. Based on the traditional Canny operator, the improved Canny algorithm employs iterative algorithm [8-9] to calculate dual threshold and to refine it by the means of mathematical morphology [10-12]. This paper's improved method of image edge detection contains the advantages of flexible 
choosing scales, rich image detail and outline information, and strong noise suppression ability.

\section{The improved Canny Edge Detection Algorithm}

The improved Canny edge detection algorithm is a method that applies iterative algorithm to calculate the optimal threshold value. This method refines the detected image edge through mathematical morphology. By analyzing and calculating the grey value of each pixel, it can effectively reduce the interference of noise by deciding whether the pixel is edge point.

The detailed procedures of the improved Canny edge detection algorithm are as follows:

(1) Process the image smoothly, by employing Gaussian filter to conduct smooth inhibition;

(2) Calculate the gradient amplitude and direction of the smoothly processed image;

(3) Conduct non-maximum suppression on gradient;

(4) Employ iterative algorithm to calculate the highest and lowest threshold;

(5) Employ double threshold algorithm to detect and connect the edge;

(6) Employ mathematical morphology method to define the edge.

The improved Canny edge detection algorithm effectively makes up for the deficiency that traditional Canny operator results in edge detection being not precise enough due to its manually set double threshold, which effectively improves the removing false edge and suppressing noise abilities of Canny operator.

\section{The Improved Algorithm of this Paper}

Wavelet transform has the character of multi-scale, making it detect by employing different methods under different scales. This paper provides a new edge detection method which based on wavelet transform and image edge detection algorithm by advanced Canny operator fusion. The advantages of the improved image edge detection method in this paper have: flexible choosing scales, rich image detail and outline information, and strong noise suppression ability.

\subsection{The Basic Principle of Improved Algorithm}

This method bases on the wavelet transform and image edge detection algorithm by advanced Canny operator fusion. Firstly, it processes the original image with the wavelet noise cancellation and smooth filtering. Then, it carry the edge detection by employing wavelet transform modulus maxima edge agglutination detection method and improved Canny edge detection method respectively to acquire their respective edge detection image. After that, it conducts wavelet fusion on these edge detection images. The fusion method that this improved algorithm adopted is: while the coefficient of low frequency is calculated though the average of both, the high frequency coefficient is acquired by the large absolute value of the wavelet coefficient, and then conduct wavelet inverse transform to refactor fusion image. 


\subsection{The Concrete Implementation Steps of the Improved Algorithm}

The diagram of the improved algorithm is shown in figure 1.

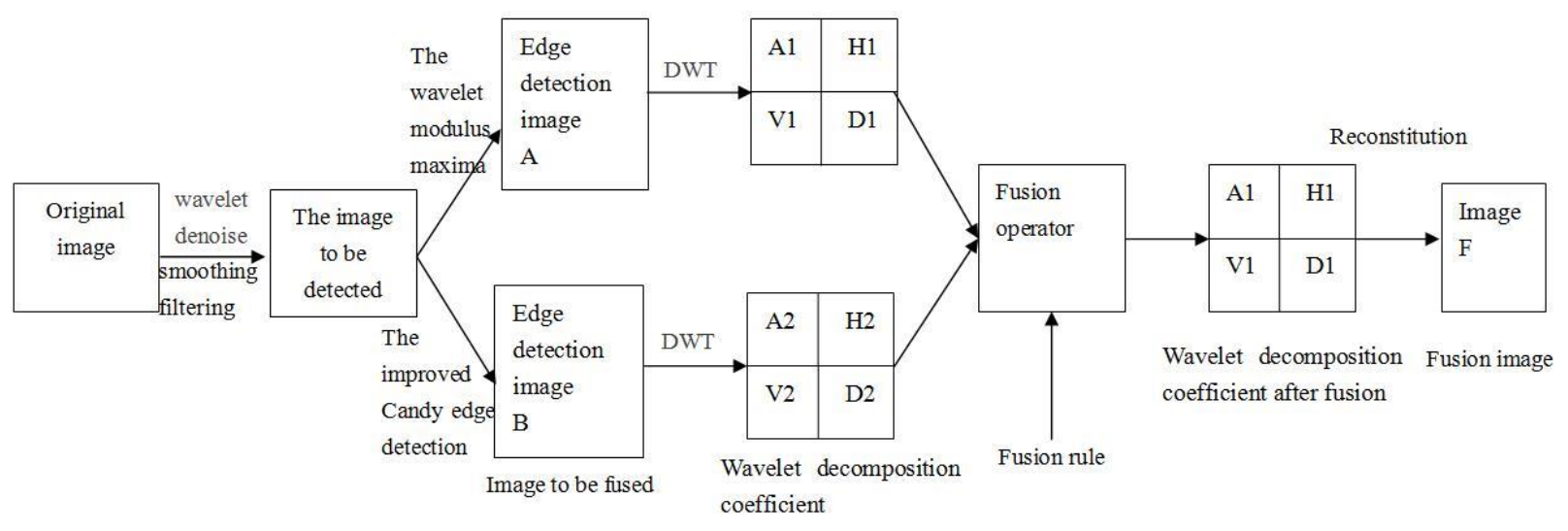

Figure 1. The Improved Algorithm Block Diagram

Its implementation steps are as follows.

(1) Employ sym4 wavelet to conduct de-noising process on the original image, and the conduct smooth filtering.

(2) Employ improved Canny operator to conduct edge detection on the image after denoising and filtering: (1) process the image smoothly, by employing Gaussian filter to conduct smooth inhibition; (2) calculate the gradient amplitude and direction of the smoothly processed image; (3) conduct non-maximum suppression on gradient; (4) employ iterative algorithm to calculate the highest and lowest threshold; (5) employ double threshold algorithm to detect and connect the edge; (6) employ mathematical morphology method to define the edge.

(3) Employ wavelet transform modulus maxima edge detection method to conduct edge detection on the image after denoising and filtering: (1) under 4 scale, employ the two-dimensional function convolution method to respectively calculate the twodimensional wavelet transform coefficients in both horizontal and vertical directions; (2) calculate the gradient amplitude and argument for each point; (3) decide which direction is the closest to the acquired angle's size and divide the argument into horizontal, vertical, positive diagonal and negative diagonal these four directions ; (4) traverse each pixel and decide whether the amplitude of the nearest direction in the corresponding angle is the maximum value, and acquire the modulus maximum points as the candidate edge points; (5) remove the small modulus maxima edge points among the candidate edge point through the threshold; (6) combine modulus maxima points under all scales to acquire the final wavelet modulus maxima edge points.

(4) Employ the wavelet fusion algorithm to fuse the two edge detection images under step (2) and step (3): (1) wavelet decomposition. Employ Haar wavelet to conduct four layered wavelet composition on fusion image, acquiring the high and low frequency information; (2) fusion. Fusion method: while the coefficient of low frequency is calculated though the average of both, the high frequency coefficient is acquired by the large absolute value of wavelet coefficients; (3) refactoring. Apply wavelet inverse 
transform method to reconstruct the image, acquiring the fusion image, that is, the final edge detection image.

\subsection{Simulation and Experimental Analysis of Improved Algorithm}

The subjects for medical images of red blood cells image $(256 \times 256)$ and Escherichia coli image $(256 \times 256)$. In Matlab2009b (7.9), respectively apply the wavelet modulus maxima edge method, improved Canny edge techniques and improved algorithm in this section to add and not add noise image for edge detection. In figure 2 and figure 3 below, we can get the simulation results.

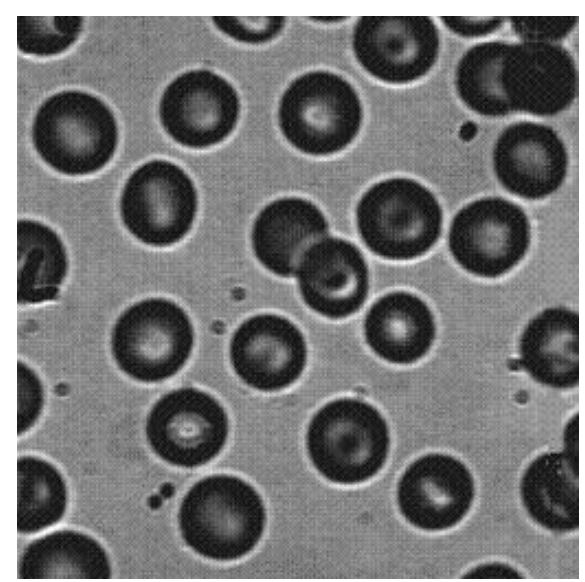

(a1) red blood cell image without noise

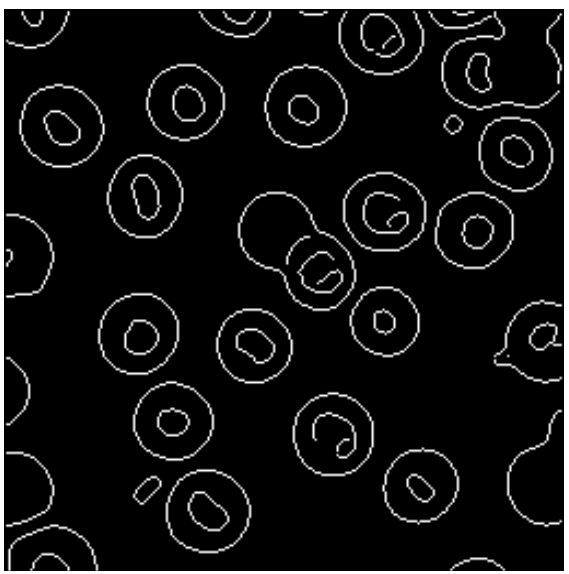

(c1) the improved Candy edge detection image



(b1) the wavelet modulus maxima edge detection

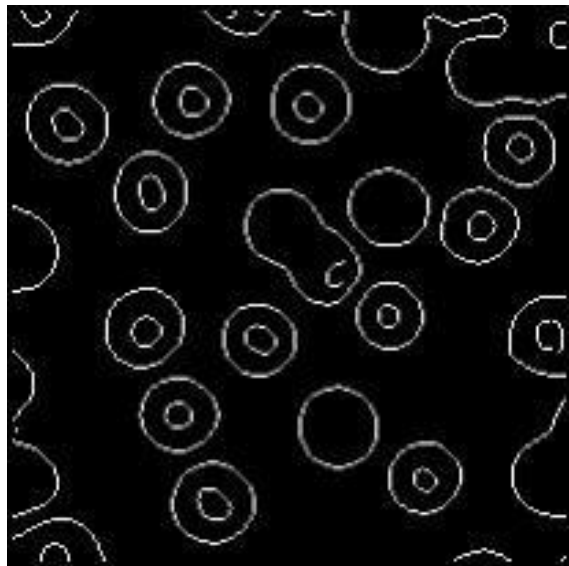

(d1) the improved algorithm of this paper image 


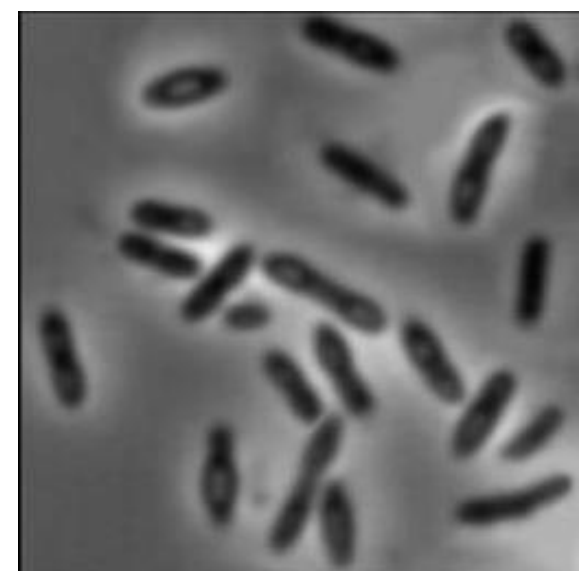

(e1) the escherichia coli image without noise

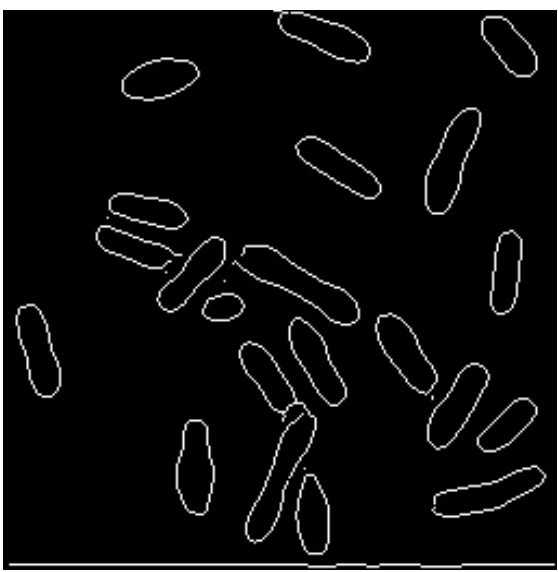

(g1) the improved Candy edge detection image



(f1) the wavelet modulus maxima edge detection

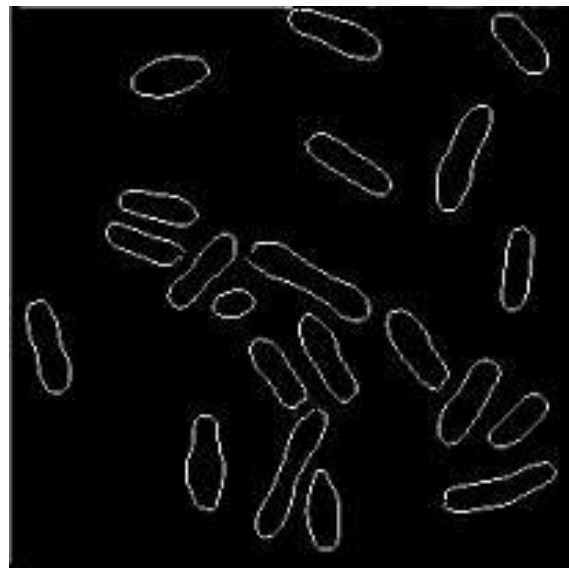

(h1) the improved algorithm of this paper image

\section{Figure 2. The Edge Detection Figure of Kinds of Algorithm without Noise}

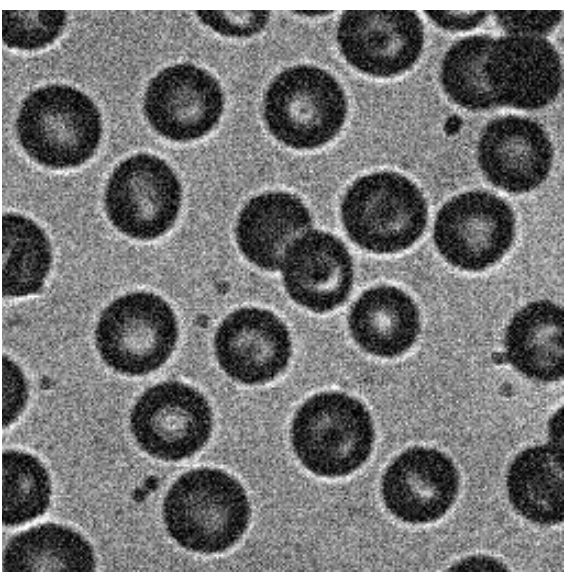

(a2) red blood cell image with noise

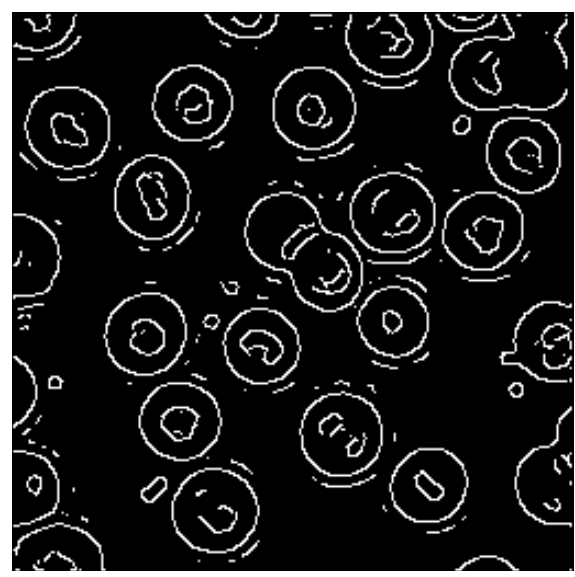

(b2) the wavelet modulus maxima edge detection 


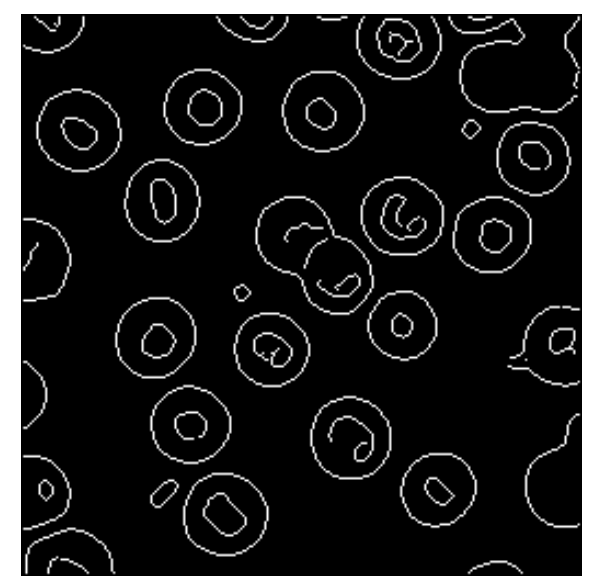

(c2) the improved Candy edge detection image

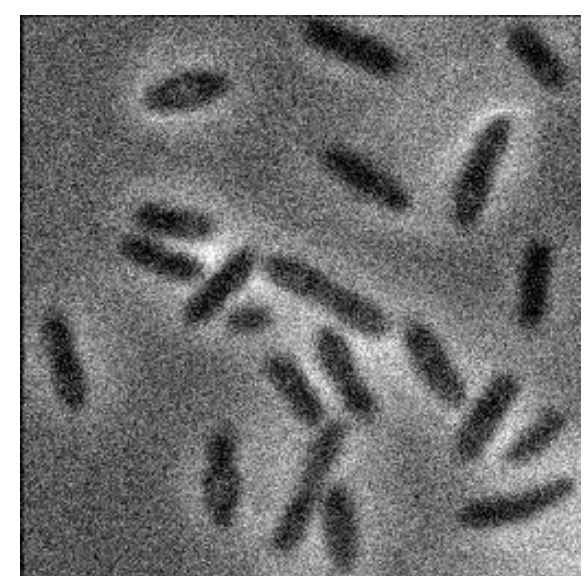

(e2) the escherichia coli image with noise

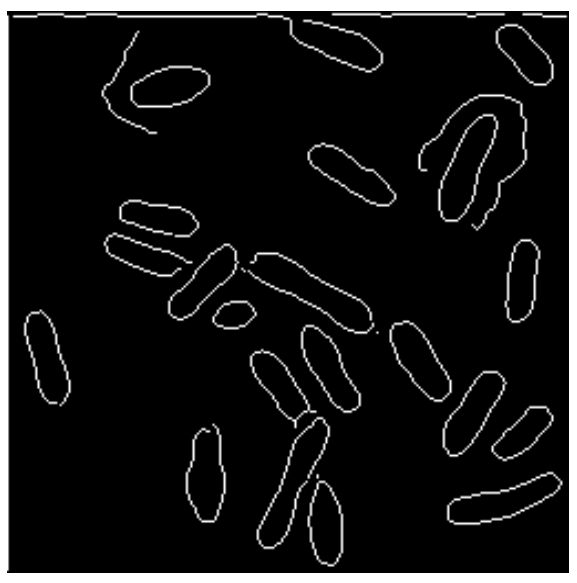

(g2) the improved Candy edge detection image

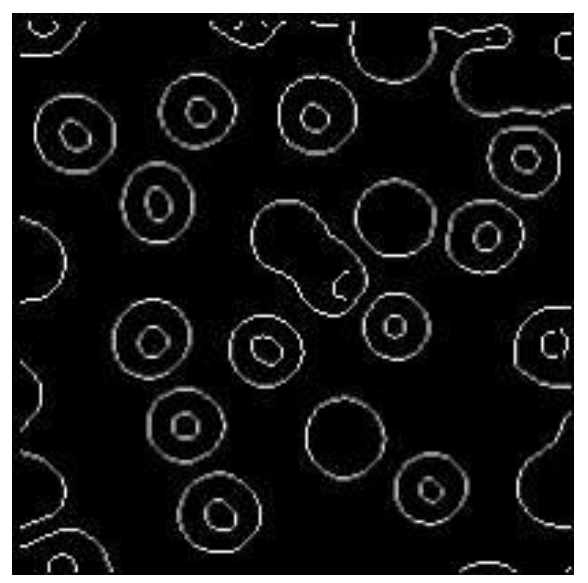

(d2) the improved algorithm of this paper image

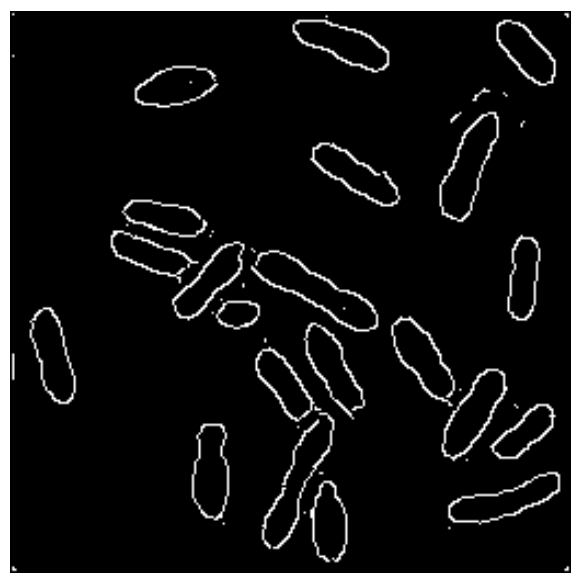

(f2) the wavelet modulus maxima edge detection

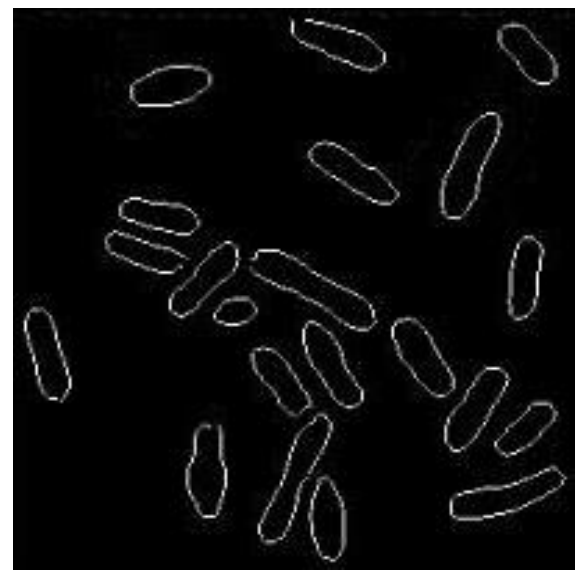

(h2) the improved algorithm of this paper image

Figure 3. The Edge Detection Figure of Kinds of Algorithm with Noise 
Observed from figure 2 and figure 3 , in this paper, the algorithm effectively combines the advantage of both wavelet modulus maxima edge techniques and improved Canny edge algorithm, and wavelet noise removal and smoothing processing to the original image. The improved algorithm not only can suppress noise effectively, but also improve the precision of edge location and its accuracy, detecting the continuous and clear edge images. The experiments proved that the method this paper proposed is an effective method for edge detection.

\section{Conclusion}

This paper provides an improved edge detection method on the wavelet fusion technology through combining the wavelet transform modulus maxima edge agglutination detection method with improved Canny edge detection method. And its experiments prove that the method greatly develops the aspects of improving edge positioning precision and suppressing noise. This paper's improved method in contrast to improved Canny operator, the improved Canny operator will cost less time, but it is not strong enough to suppress noise, while the improved algorithm in this paper is strong in noise suppression but it cost more time. Therefore, we should choose different detection algorithm according to its requirements on edge detection accuracy and time in actual application. However, how to shorten the paper's algorithm time deserves further researching.

\section{Acknowledgement}

This research was supported by the National Natural Science Foundation of China General Projects Grant No. 61272029.

\section{References}

[1] P. Melin, C. I. Gonzalez and J. R. Castro, "Edge-detection method for image processing based on generalized type-2 fuzzy logic. Fuzzy Systems", IEEE Transactions on vol. 22, no. 6, (2014), pp. 1515-1525.

[2] P. Dollár and C. L. Zitnick, "Fast edge detection using structured forests. Pattern Analysis and Machine Intelligence", IEEE Transactions on vol. 37, no. 8, (2015), pp. 1558-1570.

[3] F. Baselice, G Ferraioli and D. Reale, "Edge detection using real and imaginary decomposition of SAR data", Geoscience and Remote Sensing, IEEE Transactions on vol. 52, no. 7, (2014), pp. 3833-3842.

[4] T. Deserno, T. Aach, K. Amunts, W. Hillen, T. Kuhlen and Ingrid Scholl, "Advances in medical image processing", Computer Science - Research and Development, vol. 26, no. 1, (2011), pp. 1-3.

[5] S. Mallat and S. Zhong, "Characterization of signal from multiscale edge", IEEE Trans PAMI, vol. 14, no. 7, (1992), pp. 710-732.

[6] L. Peñailillo, R. Silvestre and K.Nosaka, "Changes in surface EMG assessed by discrete wavelet transform during maximal isometric voluntary contractions following supramaximal cycling", European Journal of Applied Physiology, vol. 113, no. 4, (2013), pp. 895-904.

[7] J. Canny, "A computational approach to edge detection. Pattern Analysis and Machine Intelligence", IEEE Transactions on, vol. 8, no. 6, (1986),pp. 679-698.

[8] X. Wang, X.Liu and Y. Guan, "Image edge detection algorithm based on improved Canny operator", computer engineering, vol. 38, no. 14, (2012), pp. 196-198.

[9] D. Li and J. Qian, "Improvement of image edge detection technology. Computer Engineering and Applications", vol. 46, no. 18, (2010), pp. 164-166.

[10] S. Mukhopadhyay and B. Chanda, "Multiscale morphological segmentation of gray scale images", IEEE Trans on Image Processing, vol. 12, no. 5, (2003), pp. 533-549.

[11] B. A. Suleimanov and O. A. Dyshin, "Application of discrete wavelet transform to the solution of boundary value problems for quasi-linear parabolic equations", Applied Mathematics and Computation, vol. 219, no. 12, (2013), pp. $7036-7047$. 
[12] S. Mallat and W. L. Hwang, "Sigularity detection and processing with wavelets", IEEE Trans IT, vol. 38, no. 2, (1992), pp. 617-643.

\section{Authors}

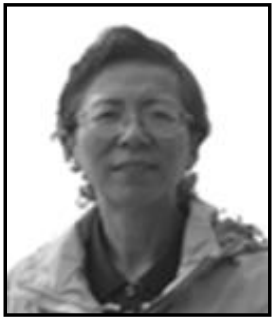

Liu Xumin, was born in Liaoning, China in 1956. She took the B.S degree in computer application, and M.E. degree in computer application from the Dalian University of Technology, in 1982 and 1993, respectively. She received the Ph.D. degree in School of Computer and Information Technology from the Beijing Jiaotong University in 2008. Currently, she is a professor at College of information Engineering, Capital Normal University. Her research

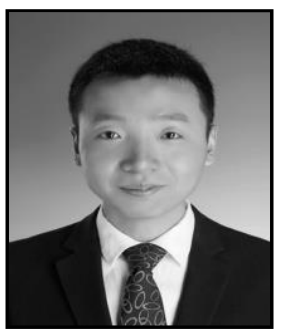

Duan Zilong, was born in handan, Henbei in 1989. He received the B.S degree in Electronic Information Engineering from North China Institute of Science and Technology, in 2012 and M.S degree in Communication Engineering from Capital Normal University, Beijing, in 2015. His research interests include digital image processing, data mining, image processing and computer graphics.

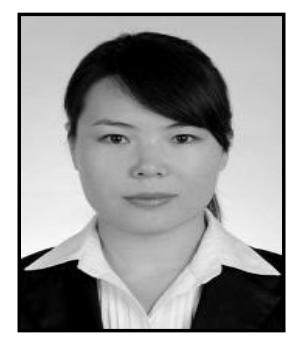

Wang Xiaojun, was born in xuchang, Henan in 1986. She received the B.S degree in Electronic Information Engineering from Henan Normal University, Henan, in 2008 and M.S degree in Communication Engineering from Capital Normal University, Beijing, in 2013. Her research interests include digital image processing, data mining, image processing and computer graphics.

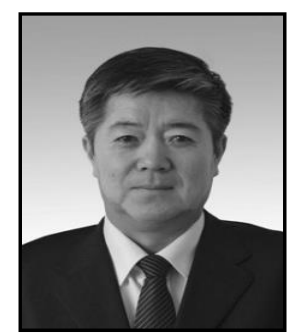

Xu Weixiang, is presently a Professor of School of Traffic and Transportation at Beijing Jiaotong University of China. He obtained his M.S degree in computer science from Dalian University of Technology of China in 1993 and $\mathrm{PhD}$ degree in System Engineering from Northern Jiaotong University of China in 2000. His current research interests focus on data mining, intelligent information processing and management, the analysis and integration for Transport systems, and their joint applications in engineering design, Rail and urban rail transit for knowledge discovery and management purpose. 